\title{
Dental caries, contact with dental services and deprivation in young children: their relationship at a small area level
}

\author{
M. Tickle, ' G. Moulding, ${ }^{2}$ K. Milsom, ${ }^{3}$ and A. Blinkhorn, ${ }^{4}$
}

Objective To measure the relationship between tooth decay, contact with dental services and deprivation at electoral ward level.

Setting The study was carried out in 1998 in Ellesmere Port in the North West of England.

Subjects and Materials All children younger than six years resident in Ellesmere Port registered with GDS services and those using CDS services were matched against the HA population register to identify unregistered children. Rates for children aged 3-5 years 'in contact' with primary dental care services, whether CDS or GDS, were calculated at ward level. One calibrated examiner examined all 5-year-old children in Ellesmere Port and $\mathrm{dmft}$ scores were calculated at ward level. Ward deprivation was measured using the Jarman score. Bivariate linear regressions at ward level were performed in turn between: $\mathrm{dmft}$ and Jarman score; rates for 3-5-year-olds in contact with dental services and Jarman score; and dmft and rates for 3-5-year-olds in contact with dental services.

Results A significant linear relationship was observed between $\mathrm{dmft}$ and Jarman score $(\mathrm{P}=0.02, \mathrm{R} 2=0.43)$. Significant inverse relationships were found between rates for 3-5-year-olds in contact with dental services and Jarman score $(\mathrm{P}=0.001, \mathrm{R} 2=$ $0.67)$, and also between $\mathrm{dmft}$ and rates for 3-5-year-olds in contact with dental services $(\mathrm{P}=0.002, \mathrm{R} 2=0.65)$.

Conclusions A strong inverse relationship was found between dental caries and contact with primary dental care services at electoral ward level. This relationship needs to be explored over a wider geographical area to establish if it is consistent and independent of deprivation.

$\mathrm{U}$ nderstanding the relationship between use of dental services and dental disease is important, as regular attendance at a dentist is advocated to promote oral health. ${ }^{1}$ The NHS is limited in the measurement of regular attendance. In the General Dental Services (GDS) we have the concept of registration, ${ }^{2}$ which is an imperfect

$1^{*}$ Senior Lecturer/Consultant in Dental Public Health, Manchester and Salford \& Trafford Health Authorities, Turner Dental School, University of Manchester ${ }^{2}$ General Dental Practitioner, Ellesmere Port PDS Consortium, Pooltown Dental Practice, Ellesmere Port ${ }^{3}$ Consultant in Dental Public Health, Chester and Halton Community Trust, Countess of Chester Health Park, Chester ${ }^{4}$ Professor of Oral Health \& Development, Turner School of Dentistry, University of Manchester ${ }^{*}$ Correspondence to: $M$. Tickle, Manchester Health Authority, Gateway House, Piccadilly South, Manchester, M60 7LP.

e-mail:Martin.Tickle@mchester-ha.nwest.nhs.uk

REFEREED PAPER

Received 24.08.99; accepted 02.02.00

(C) British Dental Journal 2000; 189: 376-379 measure of regular attendance. ${ }^{3}$ However the Department of Health considers registration to be important. It is now one of the high level indicators on which the performance of health authorities is judged, ${ }^{4}$ and general dental practitioners (GDPs) practising in disadvantaged areas receive additional payments to register children under 5 years old. ${ }^{5}$

Whether or not registration with a GDP has positive benefits for population oral health is open to debate. Studies have been equivocal; some have suggested that registration has little effect on the oral health of the individual, ${ }^{6}$ others suggest that it is beneficial. ${ }^{7}$ However most studies on registration have been performed on samples of the population and not by constructing rates at a small area level. The ability to look at registration at a small area level is important to enable targeting of schemes to promote registration. To do this, not only do the registered patients have to be identified, but also the unregistered residents have to be accounted for. Tickle et al. ${ }^{8}$ have demonstrated that it is possible to successfully identify unregistered patients by comparing registration data provided by the Dental Practice Board (DPB) with the Health Authority (HA) population register. However other factors must also be considered when constructing small area registration rates. The Community Dental Service (CDS) still has a primary care role, especially for young children. Waplington et al. ${ }^{9}$ found that approximately $9 \%$ of children aged 8 used the CDS for their dental care. Therefore the CDS can have a considerable impact on the dental service uptake of child populations and their patients must be taken into account to ensure that the number of unregistered patients is not inflated.

If the population in contact with primary dental care is to be related to population dental caries experience, deprivation must

Table I Results of three bivariate linear regressions models for the relationships at ward level

Regressions are between

- dmft of 5-year-old children and deprivation as measured by the Jarman index

- service contact rate per 100 population for 3-5 year-olds and deprivation as measured by the Jarman index

- dmft of 5-year-old children and service contact rate per 100 population for 3-5 year-olds showing the B coefficients, their standard errors, $P$ values and the $R^{2}$ statistics

\begin{tabular}{|c|c|c|c|c|c|}
\hline Dependent variable & $\begin{array}{l}\text { Independent } \\
\text { variable }\end{array}$ & B & B (SE) & $\boldsymbol{P}$ & $\mathbf{R}^{2}$ \\
\hline $\mathrm{dmft}$ & Jarman score & 0.012 & 0.004 & 0.02 & 0.43 \\
\hline $\begin{array}{l}\text { Service contact rate for } \\
3-5 \text { year-olds }\end{array}$ & Jarman score & -0.245 & 0.054 & 0.001 & 0.67 \\
\hline $\mathrm{dmft}$ & $\begin{array}{l}\text { Service contact } \\
\text { rate for 3-5 } \\
\text { year-olds }\end{array}$ & -0.056 & 0.013 & 0.002 & 0.65 \\
\hline
\end{tabular}


also be considered. Dental caries is positively associated with deprivation, ${ }^{10,11}$ but deprivation also has a significant inverse relationship with dental service use $^{3}$ and registration. ${ }^{7}$ Therefore socio-economic status can act as a confounder when examining the relationship between service use and dental disease.

With an ability to match dental data sets to the Health Authority (HA) register, the relationship between tooth decay and contact with dental services at a small area level should be apparent. Therefore the objectives of this study were to construct rates for children 3-5-years-old 'in contact' with primary dental care from data provided by the DPB and CDS at electoral ward level for a specific locality, and then compare these rates with ward deprivation rates and $\mathrm{dmft}$ scores.

\section{Method}

The study took place in the town of Ellesmere Port, South Cheshire, which is a geographically discrete community. The total mid-year population estimate in 1997 of Ellesmere Port Borough (including the villages of Neston and Willaston) was 85,387. Ellesmere Port is the site of a first wave Personal Dental Services (PDS) pilot in which all 21 GDPs practising in the town are working collectively to provide a primary dental care service. The PDS pilots started in October 1998 and will test new models of dental care provision which are tailored to address the specific oral health or service issues of identified populations. All 21 dentists gave permission for files of their patients to be supplied by the DPB to the Health Authority. The CDS has two clinics in Ellesmere Port, which supplied information on all patients who were in treatment or awaiting recall appointments. The CDS and GDS data were read into Microsoft Access then cleaned and aggregated to make a single data file.

This dental data file was processed against the HA population register so that the unregistered patients could be identified, the methodology used has been described in detail elsewhere. ${ }^{8}$ The unregistered records were flagged and added to the dental data file. Electoral ward codes and the relevant ward Jarman deprivation scores $^{12}$ were added to the data set using the subjects' postcodes. Rates per 100 population for children aged 3-5 years 'in contact' with primary dental care services, whether CDS or GDS, were calculated at electoral ward level. Electoral wards are units of Census geography and local government democracy. The geographical and population size of wards vary according to location, however in the North West Region the mean ward population size in mid-1994 was 6,995.4

During the $1997 / 8$ NHS nationally co-ordinated epidemiological survey all 5-year-old children in Ellesmere Port were examined by one calibrated examiner. Ward codes were added to the records, again by reference to the postcode, and ward dmft scores were calculated. The data were read into SPSS and bivariate linear regressions were performed in turn between:

- Ward dmft and the ward Jarman score

- Ward rates for 3-5-year-olds in contact with dental services and the ward Jarman score

- Ward dmft and ward rates for 3-5-year-olds in contact with dental services.

Scatter plots with regression lines and their 95\% confidence intervals were also generated. The regression analyses with $\mathrm{dmft}$ as the dependent variable were weighted according to the number of children in each ward examined in the NHS survey.

\section{Results}

Ellesmere Port is made up of 12 electoral wards, which had a mean total mid-1994 Census population of 5,016 (range 285-6691). Ward Jarman scores for the locality ranged from -27.15 to 34.56 , with a mean of 5.63, six wards had positive scores and six had negative scores.

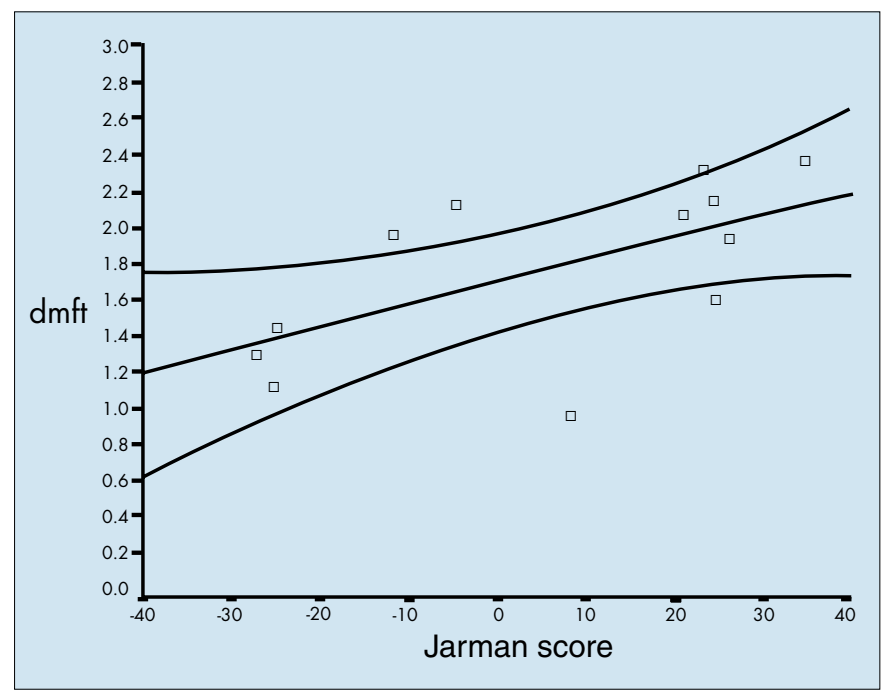

Fig. I Scatter plot and regression line with 95 percent confidence intervals of dmft of 5-year-old children and the Jarman deprivation score at electoral ward level.

A total of 1630 children aged 3-5 years were registered with a GDP practising in Ellesmere Port, and 185 children of the same age group were in treatment or awaiting recall in the CDS. Therefore 1815 children were 'in contact' with primary dental care in Ellesmere Port. Of these records 116 failed to match with the HA population register, however, 71 of these could be categorised as living outside Ellesmere Port. Therefore only 45 (2.5\%) of the dental records could have been Ellesmere Port residents but were unable to be allocated to an electoral ward. These records were excluded from the analyses. A further 1108 children aged 3-5-years were identified in the HA population register, as living in Ellesmere Port and not present in the dental data sets. These were the unregistered children. The ward of residence of all subjects on the HA register, either in contact with primary dental care or unregistered, could be identified.

The 1997/8 NHS epidemiological survey of 5-year-old children was performed at the same time as the data matching process. All 8625 -year-old children attending state school in Ellesmere Port children were examined. Out of this total only $18(0.9 \%)$ records

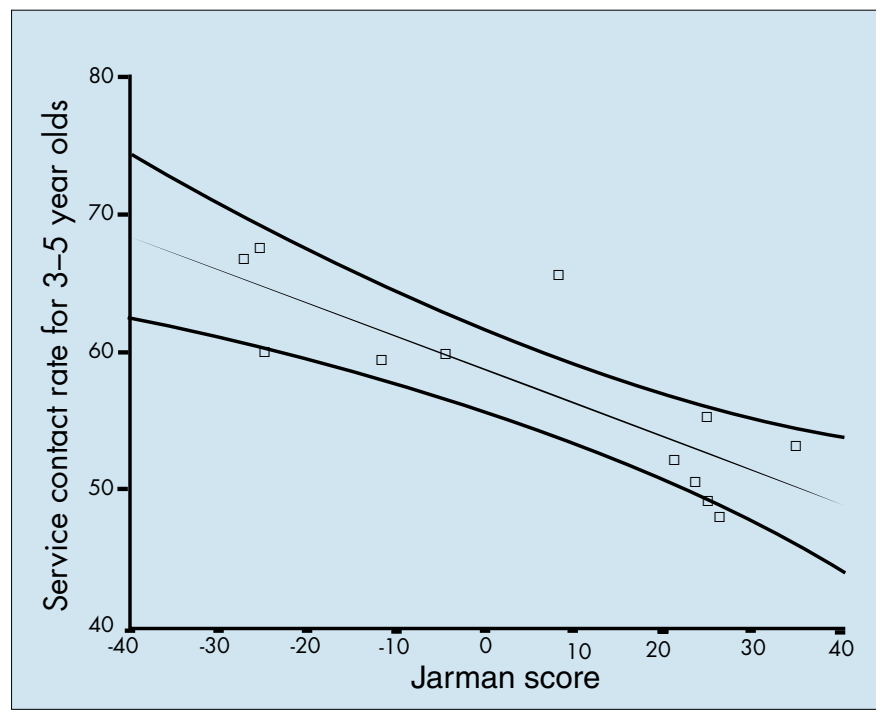

Fig. 2 Scatter plot and regression line with 95 percent confidence intervals of rates per 100 population for children aged 3-5 years in contact with primary dental care and the Jarman deprivation score at electoral ward level. 


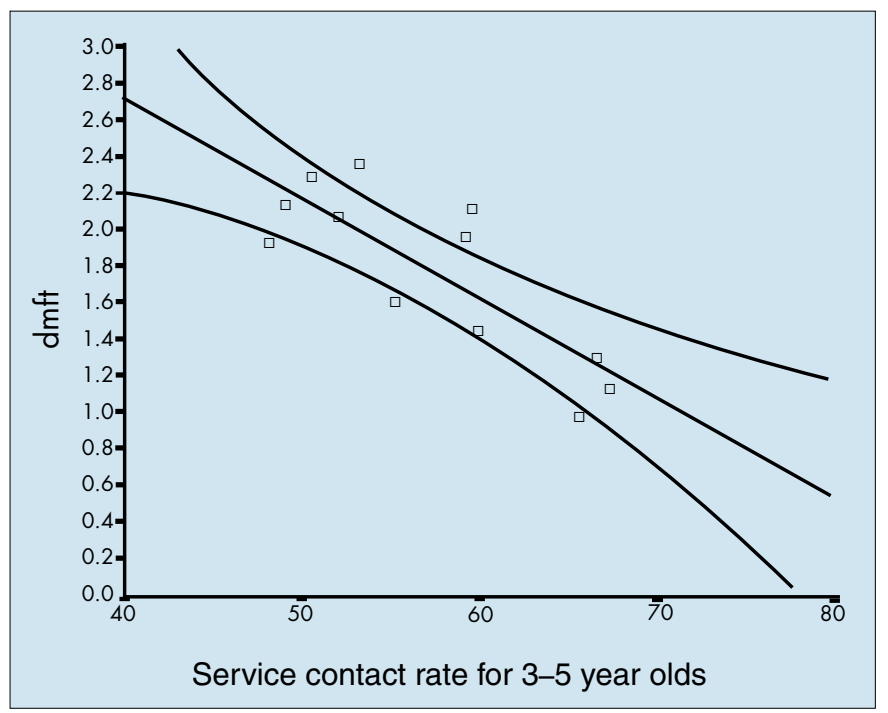

Fig. 3 Scatter plot and regression line with 95 percent confidence intervals of $\mathrm{dmft}$ of 5 -year-old children and rates per 100 population for children aged 3-5 years in contact with primary dental care at electoral ward level.

could not be assigned a ward code from their home postcode. The mean ward population size in this data set was 71.8 (range 42-137) and ward dmft ranged from $0.98-2.35$ with a mean of 1.78 (S.D. $0.46)$.

Table 1 summarised the results of the three bivariate regression analyses. Fig. 1 depicts the bivariate relationship at ward level between $\mathrm{dmft}$ as the dependent variable and Jarman score as the independent variable. There was a significant $(\mathrm{P}=0.02)$ linear relationship, and the Jarman index explained $43 \%$ of the variability of $\mathrm{dmft}$. The bivariate relationship between ward rate for contact with primary dental care for 3-5-year-olds as the dependent variable, and Jarman score as the independent variable is displayed in Figure 2 . There was a highly significant $(\mathrm{P}=0.001)$ inverse linear relationship and the Jarman score explained $67 \%$ of the variability of contact with primary dental care. Ward rates for service contact for $3-5$-year-olds also exhibited a highly significant $(\mathrm{P}=0.002)$ inverse relationship with $\mathrm{dmft}$, dental caries experience fell as service contact increased, the relationship is shown in Figure 3. Service contact for 3-5-year-olds explained $65 \%$ of the variability of $\mathrm{dmft}$ for 5 year-olds at ward level and for each unit rise in service contact, ward $\mathrm{dmft}$ decreased by 0.06 of a tooth.

\section{Discussion}

The payment system of the PDS pilot at Ellesmere Port is based on registrations, and the pilot is working to fulfil the aims of South Cheshire Health's Oral Health Strategy which has targets to reduce the mean $\mathrm{dmft}$ score in 5-year-olds. Therefore the questions under study here are particularly relevant to the GDPs working in Ellesmere Port.

The principal advantage of matching GDS and CDS data to the HA population base was that reasonably accurate rates for service contact could be calculated, and analyses at a small area-level could be undertaken. The bivariate analyses at ward level showed a significant $(\mathrm{P}=0.02)$ linear relationship between $\mathrm{dmft}$ and Jarman score. This significant relationship at ward level was expected from the literature. ${ }^{13}$ However, the Jarman score explained only $43 \%$ of the variability of ward $\mathrm{dmft}$, a much lower percentage than that reported by Jones et al. ${ }^{13}$ in non-fluoridated Salford $(\mathrm{R} 2=0.88)$. When service contact for 3-5 year olds was regressed against Jarman a highly significant inverse relationship $(\mathrm{P}=0.001)$ was found. Again this was expected from the literature. ${ }^{7,10}$ A highly significant inverse relationship between $\mathrm{dmft}$ and contact with primary dental care was also found. The relationship between ward $\mathrm{dmft}$ and the rates of 3-5-year-olds in contact with primary dental care $(\mathrm{P}=0.002, \mathrm{R} 2=0.65)$ was much stronger than that between $\mathrm{dmft}$ and Jarman score $(\mathrm{P}=0.02, \mathrm{R} 2=0.43)$.

The rather clumsy term 'in contact with dental services' has been used to encompass those registered with a GDS dentist and those in treatment or awaiting recall with the CDS. The CDS provided primary dental care for some 10.2 percent $(\mathrm{N}=185)$ of the population 'in contact' with primary dental care, and therefore could not be ignored. Therefore a decision was taken to collect information on CDS patients under treatment or awaiting recall, a situation broadly analogous to registration in the GDS. Using registration is also problematical. It has been criticised as a measure of service use, as it does not represent regular, asymptomatic attendance at the dental surgery. ${ }^{3}$ Even with these difficulties of an imperfect measure for use of dental services, the association between disease and service contact - most of which was made up of GDS registration was stronger than between disease and deprivation.

The findings reported here suggest that at the population level, service use is associated with decreased disease experience. However these results could be confounded by socioeconomic status. Tickle et al. ${ }^{3}$ in a study at the individual level showed using multiple regression that regular attendance at the dental surgery had a significant inverse relationship with $\mathrm{dmft}$ after controlling for socioeconomic status. After a huge amount of work in the matching exercise $^{8}$ service contact rates could only be calculated for the twelve wards of Ellesmere Port. Therefore the numbers were too small to support a multiple regression analysis to confirm that contact with dental services had an independent association with $\mathrm{dmft}$ after controlling for deprivation. The small numbers also explain the wide confidence intervals evident in each of the scatter plots.

The strong inverse relationship found between $\mathrm{dmft}$ and contact with primary dental care is important. It could be that the association found could be attributed to dentists providing preventive advice and care to their patients. However, the strong relationship found between disease and service use does not signify cause and effect. It does not follow from these data that by increasing population registration rates $\mathrm{dmft}$ will fall. From a public health view we need evidence to understand whether specific programmes to increase registration are effective in reducing population disease experience.

The relationship found could also be explained by the fact that accessing dental services forms part of a group of associated behaviours ${ }^{14,15}$ aimed at minimising risk of developing dental disease. If this is the case a measure of regular contact with primary dental care, together with measures of deprivation, could increase the accuracy of predictive models to provide estimates of population caries experience at a small area level. This could reduce the need for large and costly epidemiological surveys to measure population disease levels. To test these questions and to test whether or not the results found in Ellesmere Port are generalisable across the UK, health service researchers need wider access to the data sets held by the DPB and CDS, which represent a potentially valuable but under-used NHS resource. A geographically broader based study would also provide more scatter plot points and firmly establish the nature of the relationship between $\mathrm{dmft}$ and service contact.

As the PDS pilots, and now Dental Access Centres progress registration in its present form may occupy a less prominent place in NHS policy, and it may be more appropriate for the profession to agree on a standardised measure of regular, asymptomatic attendance to be collected across primary dental care. Such an approach would perhaps be a better indicator of preventive behaviour and potentially a more powerful predictor of population disease. There are many technical, ethical and economic barriers to overcome in order to meet these objectives and there will have to be the political 
will to support these developments. We can only hope that these issues will be addressed in an information management and technology component to the new strategy for dentistry.

In conclusion a strong inverse relationship between dental caries and contact with primary dental care services was found at ward level. This relationship needs to be explored over a wider geographical area to establish if it is consistent and independent of deprivation. In the longer term the reasons for this relationship should be investigated in order to establish an evidence-base for the advocacy of regular dental attendance.

1 Levine R. The Scientific Basis of Dental Health Education. 4th ed. 1996 London, Health Education Authority

2 Department of Health The Dental Contract detailed guidance Annex to FPN 5241990 HMSO, London

3 Tickle M, Williams M J, Jenner A M and Blinkhorn A S. The effects of dental attendance and socio-economic status on dental caries experience and treatment patterns in 5-year-old children Br Dent J 1999;186; 135-137

4 Department of Health The new NHS Modern and Dependable: A national framework for assessing performance consultation document EL (98) 4, 1998 London, HMSO

5 Department of Health General Dental Services HSC 1998/042, 1998 London, HMSO.

6 Daley F M, Milsom K M and Lennon M A. The relationship between reg- istration and dental health in 8 and 9-year-old children in Cheshire $\mathrm{Br}$ Dent J 1994; 177: 416-418

7 White D and Anderson R J. Children's dental health under the capitation scheme Community Dent Health 1996; 13 (Suppl): 21-48

8 Tickle M, Hayhurst G, Moulding G, Jenner A M, Blinkhorn A S. Matching Dental Practice Board data to the Health Authority Population Register Community Dent Health (In press)

9 Waplington J A, White D A and Clarke J R. A comparison of the social backgrounds and dental health of patients attending the Community Dental Service, the General Dental Service and non registered patients. Community Dent Health 1998; 15: 150-154

10 Silver D H. A comparison of 3-year-old's caries experience in 1973, 1981, and 1989 in a Hertfordshire town, related to family behaviour and social class Br Dent J 1992; 172, 191-197

11 Hinds K, Gregory J. National Diet and Nutrition Survey: Children aged $11 / 2$ to $41 / 2$ years. 1995 Volume 2 report of Dental Survey HMSO, London 12 Jarman B. Identification of underprivileged areas. BMJ, 1983; 286: 17051709.

13 Jones C M, Taylor G O Whittle J G Evans D and Trotter D P. Water fluoridation, tooth decay in 5 year olds, and social deprivation measured by the Jarman score: an analysis of data from British dental surveys. BMJ 1997; 315: 514-7

14 Blinkhorn A S. Factors influencing the transmission of toothbrushing routine by mothers to their pre-school children. J Dent 1980; 8: 307-11

15 Roeters J, Burgersdijk R, Truin G, van 't Hof. Dental caries and its determinants in 2 to 5-year-old children J Dent Children 1995; 401-408 